Available online at GSC Online Press Directory

GSC Biological and Pharmaceutical Sciences

e-ISSN: 2581-3250, CODEN (USA): GBPSC2

Journal homepage: https://www.gsconlinepress.com/journals/gscbps

(REVIEW ARTICLE)

\title{
Exploration of vulnerability in neurodegenerative diseases: Determination skills of genetic and environmental risk factors
}

\author{
Bonnie Welch, Diane E Heck and Hong Duck Kim* \\ Department of Public Health, School of Health Sciences and Practice, New York Medical College, Valhalla NY.
}

Publication history: Received on 16 July 2020; revised on 03 August 2020; accepted on 12 August 2020

Article DOI: https://doi.org/10.30574/gscbps.2020.12.2.0226

\begin{abstract}
Neurodegenerative diseases (ND) affect a large portion of our elder population with cognitive impairment, impaired movement due to loss of balance or muscle weakness in motor neuron. Due to their irreversible nature they present a significant public health burden through the demand on our health care system as well as by unmet therapeutic measures that influence socioeconomic decision processes, while our current population is transitioned to an aged community. Here we introduce top 3 of the most prevalent NDs and examine preventive alternatives along with the available research on both genetic and environmental risk factors. While many questions remain, genetic predisposition combined with environmental exposures may be to blame for a large number of these cases. Utilizing emerging multifold omics technologies, early prevention aided by development of biomarker can help to identify risk factors and to envision new avenue in new treatments option in the future.
\end{abstract}

Keywords: CNS diseases; Alzheimer's disease (AD); Parkinson's disease (PD); Amyotrophic lateral sclerosis (ALS); metabolomics; molecular based risk assessment skills; Omics

\section{Introduction}

Neurodegenerative disease (ND) describes multiple diseases which affect the central nervous system and neurons in the brain. Neurons once formed don't reproduce or repair, so damage to a neuron is permanent [1] NDs are incurable and progressive diseases that eventually lead to the death of the patient. Included in the category of NDs are diseases which mainly affect older adults (those over the age of 60) such as Alzheimer's disease, Parkinson's disease, and Amyotrophic lateral sclerosis among several others [1]. This review will examine genetic and environmental risks associated with these three.

In each of these pathologies, age has been identified as a risk factor, even though none of these diseases are considered part of the normal aging process. In addition, studies have been able to identify other environmental risk factors which we will examine. With the help of omics technologies including genomics, proteomics, transcriptomics and metabolomics, biomarkers and genetic risks have been identified for each disease. While advances in care are helping these patients to live longer, the goal is treatments that are able to cure, stop, or reverse the damage done to the neurons by these pathologies.

The purpose of this paper is to look at ways using omics as a molecular navigator can lead us closer to development of prevention methods or improved treatment of ND's.

\footnotetext{
* Corresponding author: Hong Duck Kim

Department of Public Health, School of Health Sciences and Practice, New York Medical College, Valhalla NY 


\section{Alzheimer's disease}

Alzheimer's disease (AD) is the most prevalent ND, affecting millions of Americans. One of every 10 people over the age of 65 is living with $A D$ and it is the $5^{\text {th }}$ leading cause of death for that age group [2]. AD is typically late onset but in rare cases $(<10 \%)$ patients may develop AD early [3]. By the year 2050, it is predicted we will have 13.8 million Americans living with $\mathrm{AD}$.[2] This year, the cost of Alzheimer's to our nation is estimated at $\$ 305$ billion dollars and barring treatment advances, that number is expected to rise to over $\$ 1$ trillion per year by 2050 (AA, 2020).[2] This disease is characterized by formation of amyloid plaques and tangles within the brain and deterioration of the neurons associated with memory leading to memory loss that progresses over time until the patient may even forget how to speak. A definitive diagnosis of $\mathrm{AD}$ cannot actually be given until the patient has died and brain tissue has been examined [4].

Genetic variations have been identified that are a risk factor for developing AD. APOE $\varepsilon 4$ variants on chromosome 19 are considered a risk factor for late onset as well as TREM (triggering receptor expressed on myeloid cells) variants, while early onset is associated with APP (amyloid beta precursor protein) on chromosome 21, PSEN1(presenilin 1) on chromosome14 and PSEN2 (presenilin 2) on chromosome 1 [2,3]. Metabolomics studies have given insight to the mechanisms of AD in the body and suggest that it might be more of a metabolic disorder [4].

Environmental factors that have been implicated in AD include exposure to neurotoxic metals (lead, mercury, aluminum, cadmium, and arsenic) and pesticides due to increased activity in beta-amyloid peptides and phosphorylation of the Tau protein, which lead to the formation of plaques and tangles in the brain [5]. Ambient air pollution has also been demonstrated to have a strong association with neurodegeneration [6]. Additionally, chronic stress has been shown to confer a higher risk of developing $\mathrm{AD}$, as the microglial cells which respond to stress have also been linked to beta-amyloid accumulation [7].

\section{Parkinson's disease}

Parkinson's disease (PD) similarly attacks neurons, but those associated with movement, so it results in shaking and stiffness, which gradually progresses to problems walking, balancing and with coordination [8]. It is characterized by accumulation of Lewy bodies in the brain and spinal cord and loss of dopamine secreting neurons [9]. In the US, there are about 60,000 new cases of PD each year and this year about 900,000 Americans will be living with the disease (Parkinson's Foundation, 2019) [10]. The costs associated with care for PD are estimated to be $\$ 52$ billion per year including treatment, social security payments, and lost income [10].

One study done in Greece compared 13 gene variants previously identified as PD risks in a genome-wide association study (GWAS). They concluded that 5 out of the 13 single nucleotide polymorphisms (SNP's) (rs12185268, rs6599389, rs356220, rs13312, and rs17649553) were nominally significantly associated with PD [9]. Interestingly enough, being male is also considered a genetic risk factor for developing PD [11].

The Greece study also looked at environmental factors and they found exposure to pesticides and other toxins, severe head injury, and consumption of red meats, nuts, and soft drinks all increased the risk of developing PD [9]. Another area of research for risk factors of PD is diet and nutrition. One study found a potential protective effect of uric acid, coffee, and tea in men as well as an increased risk associated with the consumption of dairy products [12].

\section{Amyotrophic lateral sclerosis}

Amyotrophic lateral sclerosis (ALS) is characterized by progressive muscle weakness eventually leading to paralysis caused by degenerating motor nerve cells in the brain and spinal cord [13]. ALS is typically fatal 2-4 years from diagnosis, but more recently due to advances in care people are living longer.[13,14] There are about 30,000 cases in the US with about 5,000 new cases each year [13]. ALS is familial in 5-10\% of cases suggesting a genetic component, however, Guam has traditionally been an area of high prevalence; at one time 50-100 times higher than the global rate, there are currently about 3 times as many cases in Guam as in the US or Europe [14].

Bozzoni et al. did a systematic review of studies evaluating environmental factors for ALS. They found strong evidence that a genetic predisposition in paraoxonase 1 (PON1) variants and that paraoxonase 2 (PON2) variants led to down regulation in the central nervous system of ALS patients, especially with long-term exposure to pesticides [14].

They also found that while heavy metals have long been postulated as a possible risk factor, only 3 out of 50 studies showed a strong correlation and no epidemiological studies showed a strong relationship [14]. There remains a 
somewhat controversial question of why there is a high prevalence of ALS among soccer players, but not other sports and there seems to be no clear answer at this time. One other possible risk factor that has been identified $\beta$ - $N$ methylamino-L-alanine (BMAA) which is a cyanotoxin that can be found in several global environments [14]. Particularly, it is found in cycad seeds, which are used to produce flour in Guam and may contribute to the higher incidence in that nation [14].

\section{Trends for future direction}

Many of these environmental risk factors are still considered "possible", so more work needs to be done to positively identify which factors play a key role. In addition to those already mentioned above insomnia has emerged as a possible risk factor [15]. While exposure to pesticides and metals are possible factors, it must be established positively which pesticides and metals pose a threat and at what level of exposure. Likewise, ambient air pollution should be further examined. If these environmental factors are indeed culprits in causing ND's, we must identify ways to limit exposure.

One genome-wide association study noticed that neurological disorders seem to be genetically distinct suggesting the etiologic pathways of the pathologies are independent [16]. Two parallel cohort studies, the Religious Order Study and the Rush Memory and Aging Project, are currently ongoing. These two studies began in 1994 and 1997 and are following participants closely in an attempt to identify the underlying causes of AD. Participants in the study have yearly physical exams and blood draws and agree to organ donation upon their deaths. Biospecimens both ante- and post-mortem of serum, plasma, iPSC, brain, spinal cord, nerve and muscle cells are being used to produce multi-layered omics data [17].

The use of metabolomics opens up the possibilities of identifying exactly what biological snapshot is happening at that moment with a patient, opening doors to personalized medicine. Another study has demonstrated that microRNA pairs were capable of differentiating ND's from controls and from each other, indicating the possibility of using microRNAbased diagnostics for detection or perhaps even brain microRNA's as biomarkers [18].

Functional screening using targeted proteomics and high-throughput ex vivo models are being used to identify targets for small molecule drug screens that will eventually lead to drug selections and human trials [17]. According to DiazOrtiz et al., this is exactly where the future of omics needs to go; focusing more on using small molecules, RNAi and CRISPR model systems to identify targets for mammalian trials [19].

\section{Conclusion}

Neurodegenerative diseases affect cognitive impairment and imbalance of movement due to dysfunction of motor neuron or neuronal cell death with interruption a significant portion of daily lives in our older adults which data trends show our population continues to aged society. In present, the public health burden of these pathologies will continue to grow due to unknown risks along with demanding unmet solution. Utilizing forward genetics, systems biology such as integrative omics platform and its strategy, we can hopefully find ways to improve risk assessment and develop early stage detection as well as prevention methods. Solid identification of environmental risk factors must be combined with improved detection so that we can develop clinical applications for treatment of these devastating diseases. There is a need for collaborative efforts between experts in different areas of molecular based determination such as metabolomics and neurogenetics to bring the large amounts of data generated by mapping risk genes and its phenotype alteration including conventional imaging approach together for improvements in the lives of these patients in combination with nutrition therapy and physical exercise.

\section{Compliance with ethical standards}

\section{Acknowledgments}

This work was supported by the Student fund in School of Health Science and Practice and Institute of Public Health, New York Medical College.

\section{Disclosure of conflict of interest}

No conflict financial authors declared. 


\section{References}

[1] EU Joint Programme-Neurodegenerative Disease (JPND). (2019). What is Neurodegenerative Disease?

[2] Alzheimer's Association (AA). (2020). Facts and Figures.

[3] National Institutes of Health (NIH). (2015). Alzheimer's disease Genetics Fact Sheet.

[4] Wilkins JM and Trushina E. (2018). Application of metabolomics in Alzheimer's disease. Frontiers in neurology, 8, 719 .

[5] Chin-Chan M, Navarro-Yepes J and Quintanilla-Vega B. (2015). Environmental pollutants as risk factors for neurodegenerative disorders: Alzheimer and Parkinson diseases. Frontiers in cellular neuroscience, 9, 124.

[6] Dimakakou E, Johnston HJ, Streftaris G and Cherrie JW. (2018). Exposure to environmental and occupational particulate air pollution as a potential contributor to neurodegeneration and diabetes: a systematic review of epidemiological research. International journal of environmental research and public health, 15(8), 1704.

[7] Bisht K, Sharma K and Tremblay MÈ. (2018). Chronic stress as a risk factor for Alzheimer's disease: Roles of microglia-mediated synaptic remodeling, inflammation, and oxidative stress. Neurobiology of stress, 9, 9-21.

[8] National Institute of Health (NIH). (2017, May 16). Parkinson's disease.

[9] Georgiou A, Demetriou CA, Christou YP, Heraclides A, Leonidou E, Loukaides P, Yiasoumi E, Pantziaris M, Kleopa K, Papacostas SS, Loizidou M, Andreas Hadjisavvas A, Loizidou MA, Hadjisavvas A and Zamba-Papanicolaou E. (2019). Genetic and environmental factors contributing to Parkinson's disease: a case-control study in the Cypriot population. Frontiers in neurology, 10, 1047.

[10] Parkinson's Foundation. (2019, June 13). Statistics.

[11] Pinares-Garcia P, Stratikopoulos M, Zagato A, Loke H and Lee J. (2018). Sex: a significant risk factor for neurodevelopmental and neurodegenerative disorders. Brain sciences, 8(8), 154.

[12] Boulos C, Yaghi N, El Hayeck R, Heraoui GN and Fakhoury-Sayegh N. (2019). Nutritional risk factors, microbiota and Parkinson's disease: what is the current evidence? Nutrients, 11(8), 1896.

[13] Johns Hopkins Medicine (JHM). (2019, June 11). ALS, Amyotrophic Lateral Sclerosis, Lou Gehrig's disease.

[14] Bozzoni V, Pansarasa O, Diamanti L, Nosari G, Cereda C and Ceroni M. (2016). Amyotrophic lateral sclerosis and environmental factors. Functional neurology, 31(1), 7.

[15] Shamim SA, Warriach ZI, Tariq MA, Rana KF and Malik BH. (2019). Insomnia: risk factor for neurodegenerative diseases. Cureus, 11(10).

[16] Anttila V, Bulik-Sullivan B, Finucane HK, Walters RK, Bras J Duncan L and Patsopoulos NA. (2018). Analysis of shared heritability in common disorders of the brain. Science, 360(6395).

[17] Bennett DA, Buchman AS, Boyle PA, Barnes LL, Wilson RS and Schneider JA. (2018). Religious orders study and rush memory and aging project. Journal of Alzheimer's disease, 64(s1), S161-S189.

[18] Sheinerman KS, Toledo JB, Tsivinsky VG, Irwin D, Grossman M, Weintraub D and Elman LB. (2017). Circulating brain-enriched microRNAs as novel biomarkers for detection and differentiation of neurodegenerative diseases. Alzheimer's research \& therapy, 9(1), 89.

[19] Diaz-Ortiz ME and Chen-Plotkin AS. (2020). Omics in neurodegenerative disease: Hope or hype? Trends in Genetics, 36(3), 152-159. 